\title{
Embolization of Arteriovenous Malformation
}

\section{Efficacy and Safety of Preoperative Embolization Followed by Surgical Resection of AVM}

\author{
H. NAGASHIMA, K. HONGO*, S. KOBAYASHI*, T. TAKAMAE*, H. OKUDERA**, \\ J.I. KOYAMA*, F. OYA*, Y. MATSUMOTO*** \\ Interventional Neuroradiology Center, Aizawa Hospital, Matsumoto; Japan \\ * Department of Neurosurgery, Shinshu University School of Medicine, Matsumoto; Japan \\ ** Department of Emergency and Disaster Medicine, Toyama Medical and Pharmaceutical University, Toyama; Japan \\ *** Department of Neuroendovascular Therapy, Kohnan Hospital, Sendai; Japan
}

Key words: arteriovenous malformation, embolization, surgery

\section{Summary}

Treatment options for cerebral arteriovenous malformation $(A V M)$ are still controversial due to the recent result of stereotactic radiosurgery and the improved result of microsurgical resection. We investigated previously treated AVM cases and discussed the efficacy and safety of preoperative embolization especially for microsurgical resection of high-grade $A V M$ in the Spetzler-Martin grading.

Efficacy of preoperative embolization was evaluated based on 126 previously treated AVM cases at Shinshu University Hospital during the last 25 years. The safety of embolization was evaluated based on our previously-embolized 58 AVM cases (91 procedures) in the last 11 years after introduction of preoperative embolization for AVM. In all 126 cases, 82 were treated before introduction of embolization and 44 were treated after introduction of embolization. In 82 cases of the pre-embolization era, 63 lesions were removed totally in $63 \mathrm{AVMs}(77 \%)$, partially resected in $11(13 \%)$ and untreated in eight $(10 \%)$. In 74 surgically removed cases, 11 (15\%) cases showed severe intra/postoperative bleeding. In 44 cases of the embolization era, lesions were removed totally in 29 AVMs (66\%), disappeared only with embolization in one (2\%), disappeared with radiosurgery in seven (16\%) and were untreated in five $(11 \%)$. In 32 surgically removed cases, only one (2\%) case showed severe intra/postoperative bleeding.

In all 58 embolized cases, 44 were surgically removed, six were treated with radiosurgery, one was eliminated with embolization alone and six were partially obliterated and followed up for their location. In 91 procedures for 58 cases, two haemorrhagic and three ischemic complications occurred, three were transient and two remained having neurological deficits.

The introduction of preoperative embolization improved the total removal rate and reduced the intra/postoperative bleeding rate in surgical removal of AVM. The total risk of embolization is low and well-designed preoperative embolization makes surgical resection safer even in high-grade AVM in the Spetzler-Martin grading.

\section{Introduction}

Until late 1980's, surgical resection of the cerebral arteriovenous malformation (AVM) was one of the most important subjects in the neurosurgical field. Since the introduction of stereotactic radiosurgery in early 1990's, main stream of AVM treatment was changed to stereotactic radiosurgery due to its low peri-surgical complication rate ${ }^{1-4}$. However, long-term follow-up after stereotactic radiosurgery on AVM resulted in 
relatively low complete obliteration rate and high treatment-related complication rate ${ }^{5-9}$.

According to the long-term results of stereotactic radiodurgery and owing to the modern technical improvement of microneurosurgery, treatment options for AVM again became controversial. The American Hart Association recommended that surgical extirpation should be considered for the low-graded AVM in the Spetzler-Martin grading and only the lesions in which surgery offers increased risk based on its anatomical feature, stereotactic radiosurgery was recommended ${ }^{10}$. It is also recommended that a combined modality approach with embolization followed by surgery for selected cases of high-graded AVM.

Authors investigated previously treated AVM cases and discussed the efficacy of preoperative embolization for surgical resection in high-graded AVM and safety of preoperative embolization.

\section{Material and Methods}

Total 126 AVM cases were treated in Shinshu University Hospital since April 1978 to March 2003. Embolization of AVM was introduced in 1992, and total 58 cases (91 procedures) were embolized in Shinshu University and its affiliated hospitals.

After introduction of embolization, all AVM cases were evaluated by both interventional neuroradiologist $(\mathrm{HN})$ and neurosurgeons $(\mathrm{KH}$, SK) before starting treatment. Surgical total resection of AVM was considered as a principle, and preoperative embolizations were considered depending on the size, location and structure of an AVM. Stereotactic radiodurgery with or without embolization was considered only in cases of unruptured small AVM located in the eloquent area.

The purpose of embolization of AVM is to simplify the haemodynamic structure by occluding the nidus in order to reduce the intranidal blood flow and to dissect the margin of nidus from adjacent eloquent area ${ }^{11}$. All embolizations were performed under local anesthesia after functional test with barbiturate and lidocain ${ }^{12}$. Embolic material was selected according to the neurosurgeon's preference.

Efficacy of preoperative embolization was evaluated based on previously treated 126 AVM cases in Shinshu University Hospital. The 126 cases were divided into two groups: the
AVM cases treated before introduction of preoperative embolization and the cases after introduction of embolization. Surgical results, complications and number of open surgeries were investigated.

Safety of embolization for AVM was evaluated based on our previously-embolized 58 AVM cases since 1992. Results and embolization-related complications were investigated in total 91 procedures.

\section{Results}

The total 126 cases treated in Shinshu University Hospital, 79 were men and 47 were women, age ranged from three to 74 years old. One hundred-five AVM removed surgically, seven treated with stereotactic radiosurgery, 13 followed-up without treatment.

Eighty-two of 126 cases were treated before introduction of embolization (pre-embolization era) and 44 cases were treated after introduction of embolization (embolization era). Spetzler\&Martin grades of pre-embolization era were I in five cases $(6 \%)$, II in $32(39 \%)$, III in $21(26 \%)$, IV in $19(23 \%)$, and $\mathrm{V}$ in five $(6 \%)$. Spetzler\&Martin grades of embolization era were I in two cases (2\%), II in $11(25 \%)$, III in $20(45 \%)$, IV in six $(14 \%)$, and $\mathrm{V}$ in six $(14 \%)$. Of 82 cases of pre-embolization era, 74 $(90 \%)$ were removed surgically and eight $(10 \%)$ were not treated and followed-up. Of 44 cases of embolization era, 31 (70\%) were removed surgically, one ( $2 \%$ ) disappeared only with embolization, seven (16\%) treated with stereotactic radiosurgery and five $(11 \%)$ were not treated.

Of 74 surgically removed cases in pre-embolization era, 63 (85\%) underwent total removal, $11(15 \%)$ resulted in partial removal and $21(28 \%)$ required multi-staged surgery. Of 31 surgically removed cases in embolization era, all cases underwent total removal and four $(13 \%)$ required multi-staged surgery.

Surgery-related complications were seen in $11(15 \%)$ cases and unexpected residual nidus was seen in nine $(12 \%)$ cases in pre-embolization era. Surgery-related complications included four intraoperative uncontrollable bleeding, four postoperative severe bleeding and three potoperative late bleeding. Surgery-related complications were seen only in one (3\%) case and unexpected residual nidus was seen in one $(3 \%)$ case in the early period of 

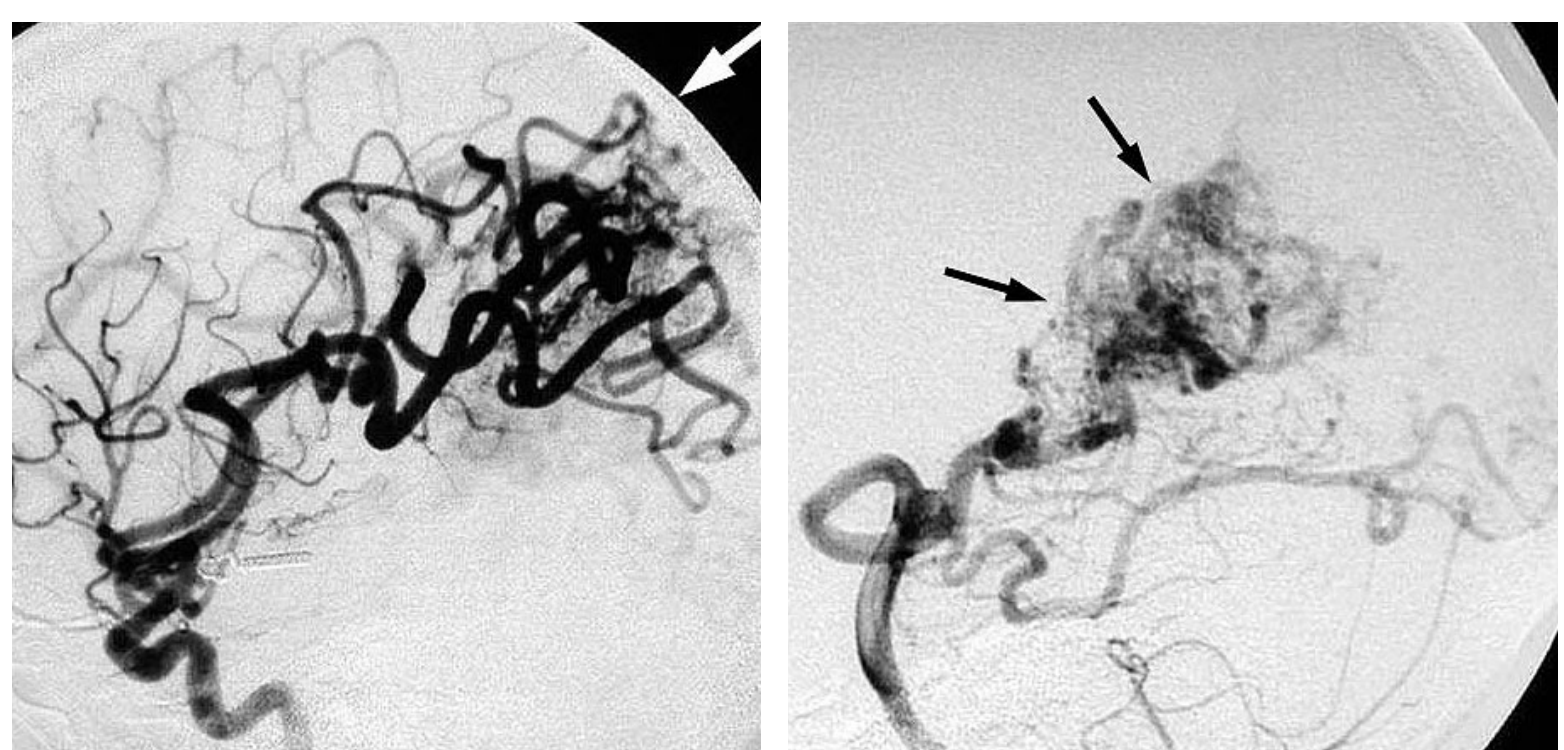

Figure 1 Pre-embolization left internal carotid (left) and vertebral (right) artery angiogram of a 24-year-old lady. Open and closed arrows indicate the margin between the nidus and normal brain.

embolization era. This surgery-related complication was intra- and postoperative severe bleeding following the incomplete preoperative embilization.

The total 58 embolized cases, 31 were men and 27 were women, age ranged from five to 76 years old. Forty-four AVM were removed, six treated with stereotactic radiosurgery, one disappeared with embolization alone and remain- ing six were observed following partial emboliztion for the deeply-seated AVM.

In 91 procedures for 58 AVM cases, the initial 43 procedures ( 26 cases) were obliteration with poly-vinyl acetate, the latter 41 procedures (30 cases) were obliteration with $\mathrm{N}$ butyl-2 cyanoacrylate (NBCA) and the remaining two cases were obliteration utilizing other materials (coil, silk) ${ }^{11,13}$.
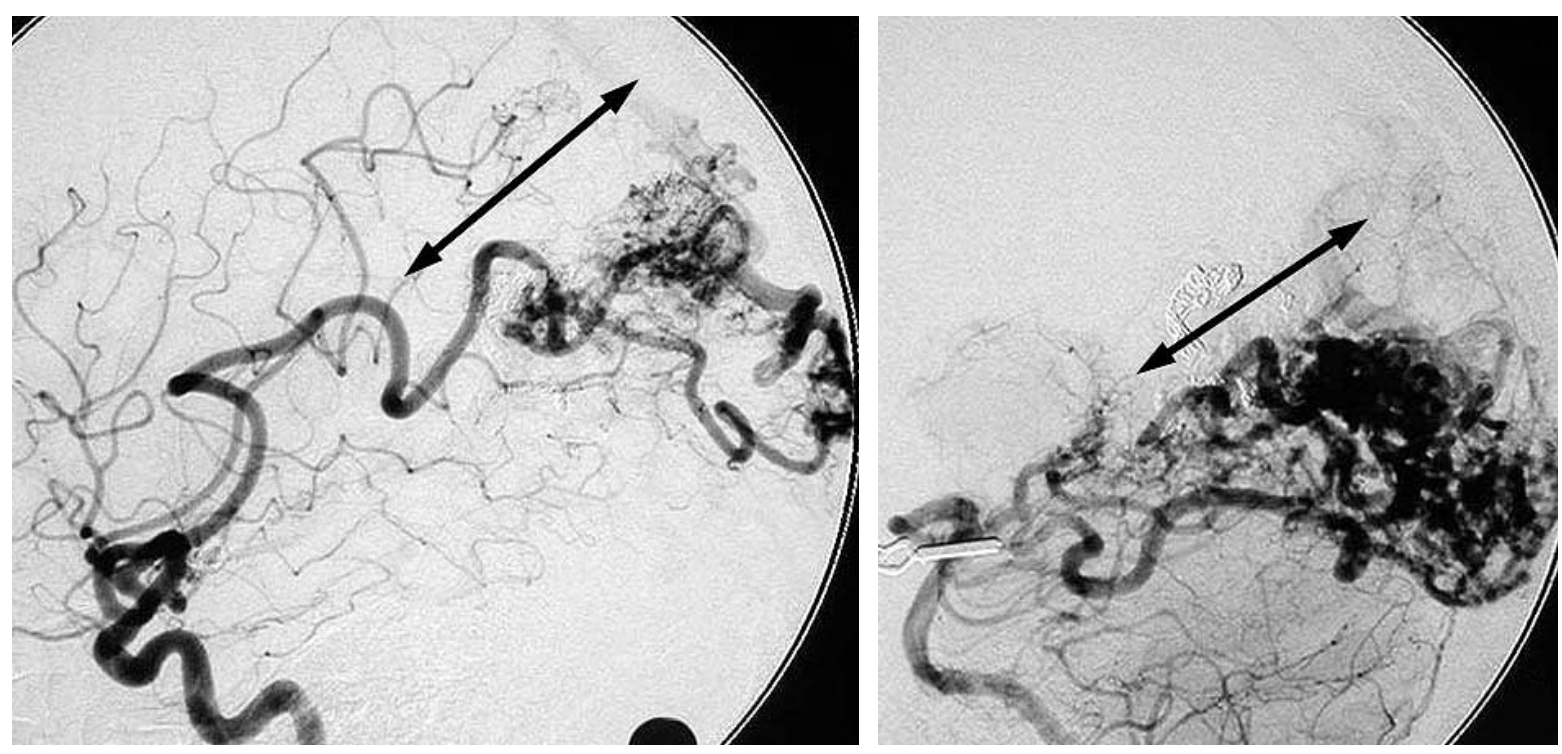

Figure 2 Post-embolization left internal carotid (left) and vertebral (right) artery angiogram of a 24-year-old lady. Arrowed lines indicate the embolized plain at the margin. 
Embolization-related complications were seen in seven cases: five were ischemic and two were haemorrhagic. In five ischemic complications, two were transient mild hemiparesis, two were permanent visual field defect and one was disconnection syndrome. The two cases which showed visual field defect were the cases with a large occipital AVM, obliterated even after positive functional test, because the symptom was prospected unavoidable after the surgical resection and the obliteration of the part judged to be benefitial to successful surgical resection. One case resulted in disconnection syndrome was the case with callosal AVM and the symptom was not detected with a functional test. In two haemorrhagic complications, one was a thin perinidal hematoma due to venous occlusion for glue migration at the end of a procedure, and the other was an intracerebellar hematoma due to rupture of a small aneurysm on the feeding artery during a procedure. The former case showed no clinical symptoms; however, the latter showed consciousness disturbance which required emergent surgical resection. Total two cases $(3 \%, 2 \%$ in total 91 procedures) showed unexpected permanent neurological deficit.

\section{Illustrative case}

This 24-year-old lady suffered sudden onset of headache. A computed tomography scan revealed an intraventricular haemorrhage and an angiogram showed a huge left occipital AVM (figure 1). After six times preoperative embolization with poly-vinyl acetate, the superiormedial and superior-lateral margin of the AVM nidus was obliterated and defended from the functioning left parietal lobe (figure 2). This AVM was successfully removed with a singlestaged surgery, and postoperatively, partial visual field deficit remained.

\section{Discussions}

Surgical resection of an AVM had a high risk of intraoperative and postoperative bleeding caused by the severe haemodynamic change ${ }^{14}$. Since the introduction of stereotactic radiosurgery, the main leading measure for AVM treatment shifted to stereotactic radiosurgery. However, long-term follow-up results of stereotactic radiosurgery for AVM showed its relatively low obliteration rate especially for a high-graded AVM, high bleeding rate before angiographical obliteration and late reappearance of the previously occluded nidus ${ }^{5-9,15,16}$.

Stereotactic radiosurgery remains an effective treatment option for treating AVM, especially for deep-seated or brainstem small lesions. However, in a low-graded superficial AVM, the indication of sterotactic radiosurgery should be discussed carefully because of its high incomplete obliteration and bleeding rate and good surgical results.

In a high-graded AVM, surgical resection also has a high risk of perisurgical complications due to the haemodynamic change. In our series of pre-embolization era, the perioperative bleeding rate was high especially in a high-graded AVM, and multi-staged resection was thus applied for avoiding this haemodynamic change ${ }^{17}$. For successful resection of a high-graded AVM, the staged preoperative embolization was introduced $^{18}$.

In our series of postembolization era showed the improved result of surgical resection in a high-graded AVM with low ( $2 \%$ for each procedure) risk accompanied with preoperative embolization. Staged and repeated preoperative embolization can reduce the flow of AVM stepwisely and reduce the risk of intraoperative severe haemodynamic change for surgical resection of a high-graded AVM. The, preoperative embolization makes haemodynamic and anatomical structures of an AVM simple. Embolization following the functional test makes the margin of the nidus possible to plain from the surrounding brain tissue especially at the eloquent area. Important matter for effective embolization prior to surgical resection is to plan the embolization procedure based on the tactics of the resection surgery and to repeat until obtaining satisfactory obliteration.

Embolization alone has a low potential for complete obliteration of $\mathrm{AVM}^{19}$ and embolization for AVM should not be planned without conjunction of other treatment.

\section{Conclusions}

The goal of treatment of an AVM is complete elimination of the opacification of a nidus. Even huge or high-graded AVMs can be safely removed by a combined-modality approach with preoperative embolization followed by surgical resection. Embolization tactics and material should be selected according to surgical strategies. 


\section{References}

1 Lunsford LD, Flickinger J, Coffey RJ: Stereotactic gamma knife radiosurgery. Initial North American experience in 207 patients. Arch Neurol 47: 169-175, 1990

2 Lunsford LD, Kondziolka D et Al: Stereotactic radiosurgery for arteriovenous malformations of the brain. J Neurosurg. 75: 512-524, 1991.

3 Steiner L, Lindquist C et Al: Clinical outcome of radiosurgery for cerebral arteriovenous malformations. J Neurosurg. 77: 1-8, 1992.

4 Friedman WA, Bova FJ: Linear accelerator radiosurgery for arteriovenous malformations. J Neurosurg 77: 832-841, 1992.

5 Karlsson B, Lindquist C, Steiner L: Effect of Gamma Knife surgery on the risk of rupture prior to AVM obliteration. Minim Invasive Neurosurg 39: 21-27, 1996.

6 Friedman WA, Bova FJ, Mendenhall WM: Linear accelerator radiosurgery for arteriovenous malformations: the relationship of size to outcome. J Neurosurg 82: 180-189, 1995.

7 Pollock BE, Flickinger JC et Al: Haemorrhage risk after stereotactic radiosurgery of cerebral arteriovenous malformations. Neurosurgery 38: 652-659, 1996.

8 Yamamoto M, Jimbo M et Al: Gamma knife radiosurgery for arteriovenous malformations: long-term follow-up results focusing on complications occurring more than 5 years after irradiation. Neurosurgery 38 : 906-914, 1996.

9 Yamamoto Y, Coffey RJ et Al: Interim report on the radiosurgical treatment of cerebral arteriovenous malformations. The influence of size, dose, time, and technical factors on obliteration rate. J Neurosurg 83: 832837, 1995.

10 Ogilvy CS, Stieg PE et Al: Special Writing Group of the Stroke Council, American Stroke Association: AHA Scientific Statement: Recommendations for the management of intracranial arteriovenous malformations: a statement for healthcare professionals from a special writing group of the Stroke Council, American Stroke Association. Stroke. 32: 1458-1471, 2001.

11 Nagashima H, Okudera H et Al: Strategic embolization for successful resection of a large cerebral arteriovenous malformation. J Clin Neurosci 7 (Suppl 1): 86-87, 2000 .
12 Sadato A, Taki W et Al: Improved provocative test for the embolization of arteriovenous malformations-technical note. Neurol Med Chir (Tokyo) 34: 187-190, 1994.

13 Ezura M, Takahashi A, Yoshimoto T: Successful treatment of an arteriovenous malformation by chemical embolization with estrogen followed by conventional radiotherapy. Neurosurgery 31: 1105-1107, 1992.

14 Tamaki N, Ehara K et Al: Cerebral hyperperfusion during surgical resection of high-flow arteriovenous malformations. Surg Neurol 40: 10-15, 1993.

15 Lindqvist M, Karlsson B et Al: Angiographic long-term follow-up data for arteriovenous malformations previously proven to be obliterated after gamma knife radiosurgery. Neurosurgery 46: 803-808, 2000.

16 Pollock BE, Kondziolka D et Al: Repeat stereotactic radiosurgery of arteriovenous malformations: factors associated with incomplete obliteration. Neurosurgery 38: 318-324, 1996.

17 Drake CG: Cerebral arteriovenous malformations: considerations for and experience with surgical treatment in 166 cases. Clin Neurosurg 26: 145-208, 1979.

18 Kinouchi H, Mizoi K et Al: Combined embolization and microsurgery for cerebral arteriovenous malformation. Neurol Med Chir (Tokyo) 42: 372-378, 2002.

19 Valavanis A, Yasargil MG: The endovascular treatment of brain arteriovenous malformations. Adv Tech Stand Neurosurg 24: 131-214, 1998.
Hisashi Nagashima, M.D. Interventional Neuroradiology Center, Aizawa Hospital, 2-5-1 Honjo, Matsumoto 390-8510, Japan 